\title{
ARTICLE
}

Clinical Study

\section{Phase 1 study of ARQ 761, a $\beta$-lapachone analogue that promotes NQO1-mediated programmed cancer cell necrosis}

\author{
David E. Gerber ${ }^{1,2,3}$, M. Shaalan Beg ${ }^{1,3}$, Farjana Fattah', Arthur E. Frankel ${ }^{1,3}$, Oluwatomilade Fatunde, ${ }^{3}$ Yull Arriaga ${ }^{1,3}$, \\ Jonathan E. Dowell ${ }^{1,3}$, Ajit Bisen ${ }^{1}$, Richard D. Leff ${ }^{4}$, Claudia C. Meek ${ }^{4}$, William C. Putnam ${ }^{4}$, Raja Reddy Kallem ${ }^{4}$, \\ Indhumathy Subramaniyan ${ }^{4}$, Ying Dong ${ }^{3}$, Joyce Bolluyt ${ }^{3}$, Venetia Sarode ${ }^{5}$, Xin Luo ${ }^{6}$, Yang Xie ${ }^{2,3,6}$, Brian Schwartz ${ }^{7}$ and \\ David A. Boothman ${ }^{3}$
}

BACKGROUND: NAD(P)H:quinone oxidoreductase 1 (NQO1) is a two-electron oxidoreductase expressed in multiple tumour types. ARQ 761 is a $\beta$-lapachone ( $\beta$-lap) analogue that exploits the unique elevation of NQO1 found in solid tumours to cause tumourspecific cell death.

METHODS: We performed a $3+3$ dose escalation study of 3 schedules (weekly, every other week, $2 / 3$ weeks) of ARQ 761 in patients with refractory advanced solid tumours. Tumour tissue was analysed for NQO1 expression. After 20 patients were analysed, enrolment was restricted to patients with NQO1-high tumours ( $H$-score $\geq 200$ ).

RESULTS: A total of 42 patients were treated. Median number of prior lines of therapy was 4 . Maximum tolerated dose was $390 \mathrm{mg} /$ $\mathrm{m}^{2}$ as a 2-h infusion every other week. Dose-limiting toxicity was anaemia. The most common treatment-related adverse events were anaemia (79\%), fatigue (45\%), hypoxia (33\%), nausea (17\%), and vomiting (17\%). Transient grade 3 hypoxia, reflecting possible methemoglobinaemia, occurred in $26 \%$ of patients. Among 32 evaluable patients, best response was stable disease ( $n=12) ; 6$ patients had tumour shrinkage. There was a trend towards improved efficacy in NQO1-high tumours $(P=0.06)$.

CONCLUSIONS: ARQ 761 has modest single-agent activity, which appears associated with tumour NQO1 expression. Principal toxicities include anaemia and possible methemoglobinaemia.

British Journal of Cancer (2018) 119:928-936; https://doi.org/10.1038/s41416-018-0278-4

\section{INTRODUCTION}

Beta-lapachone ( $\beta$-lap) is a quinone derived from the bark of South American Lapacho tree (Handroanthus impetiginosus) that exerts specific anti-tumour effects by capitalising on the increased concentration of $\mathrm{NAD}(\mathrm{P}) \mathrm{H}$ :quinone oxidoreductase 1 (NQO1) in cancer cells vs. normal cells. NQO1 is a two-electron oxidoreductase expressed in cancer tissue at levels 5 - to 200 -fold greater than in normal tissue. In cells overexpressing NQO1, $\beta$-lap initiates a futile redox cycle resulting in reactive oxygen species (ROS) generation. ${ }^{1}$ In turn, these ROS cause DNA single-strand breaks, hyperactivation of poly(ADP-ribose) polymerase-1 (PARP-1), loss of NAD+ and ATP pools, and a unique pattern of cell death referred to as "programmed necrosis" or "necroptosis". Programmed necrosis has attributes of both apoptosis (e.g. terminal deoxynucleotidyltransferase-mediated dUTP nick-end labelling positive and chromatin and nuclear condensation) and necrosis (e.g. caspase and energy independent). ${ }^{2}$
In contrast to many molecularly targeted therapies, $\beta$-lap has the potential for broad anticancer activity. Compared to normal tissue, NQO1 overexpression occurs up to 200 -fold in $>80 \%$ of non-small cell lung cancer (NSCLC), up to 100 -fold in $>80 \%$ of pancreatic cancer, up to 10 -fold in $60 \%$ of prostate cancer, up to 10 -fold in $60 \%$ of breast cancer, and up to 10 -fold in $50 \%$ of colorectal cancer. $^{3-6}$ In preclinical models, $\beta$-lap-induced cancer cell death occurs across tumours in proportion to tumour NQO1 levels. ${ }^{6-8}$ In normal tissues, induction of NQO1, a phase 2 detoxifying enzyme, occurs rarely (e.g. with substantial exposure to polyaromatic hydrocarbons) and is short-lived $(<2 \mathrm{~h})$. Importantly, factors associated with ROS detoxification (and presumptively $\beta$-lap resistance) such as catalase expression are deficient in cancer cells relative to normal tissue, further enhancing the potential therapeutic margin of this strategy in specific cancers. $\beta$-Lap has also demonstrated synergy

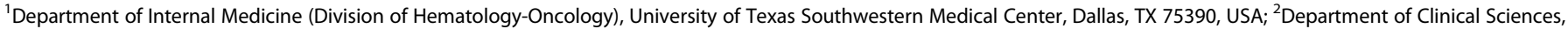

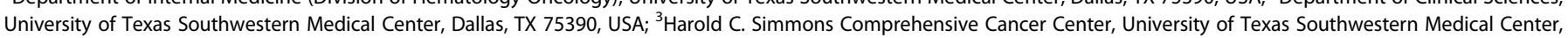

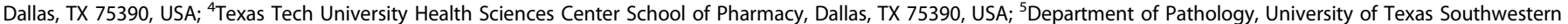

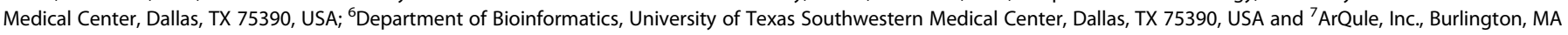
01803, USA

Correspondence: David E. Gerber (david.gerber@utsouthwestern.edu)

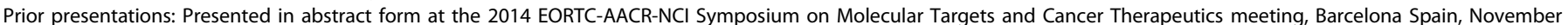
18-21, 2014, and the 2017 Annual Meeting of the American Society of Clinical Oncology, Chicago, IL, June 2-5, 2017.

ClinicalTrials.gov Identifier: NCT01502800.

Received: 3 April 2018 Revised: 23 August 2018 Accepted: 4 September 2018

Published online: 15 October 2018 
with ionising radiation, cytotoxic chemotherapy, and PARP inhibitors, ${ }^{9,10}$ rendering it potentially useful as a cancer therapy.

Despite a strong rationale and promising preclinical data, to date the clinical utility of NQO1-bioactivatable drugs has been limited. Early drugs in this class, such as mitomycin C and E09, which act by alkylating DNA, convey substantial toxicity and are susceptible to multiple resistance mechanisms, including apoptotic deficiencies and increased DNA repair. ${ }^{11,12}$ ARQ 501 (ArQule, Woburn, MA), a fully synthetic, insoluble form of $\beta$-lap that was formulated in hydroxylpropyl- $\beta$-cyclodextran (HP $\beta C D)$, advanced to single-agent and combination clinical trials in multiple cancer types. ${ }^{13,14}$ Unexpectedly, haemolytic anaemia emerged as the predominant dose-limiting toxicity (DLT) of ARQ 501. Subsequently, preclinical models suggested that ARQ 501-associated haemolytic anaemia arose from complexation with HP $\beta C D$, the carrier required for solubility, ${ }^{15}$ rather than from $\beta$-lap alone. ${ }^{16}$ Furthermore, studies of ARQ 501 did not include assessment of NQO1 biology because the drug was being developed primarily as an E2F-targeting cell cycle inhibitor. ${ }^{13,14,17}$

ARQ 761 (ArQule, Burlington, MA) is a synthetic prodrug of $\beta$-lap that is more water soluble and requires $\angle 5 \%$ of the HP $\beta C D$ required for ARQ 501 administration, thereby conveying a lower hypothetical risk of haemolytic anaemia. Upon dosing, ARQ 761 is rapidly and completely converted to ARQ 501 . We conducted a first-in-human phase 1 dose escalation trial of ARQ 761 in refractory advanced solid tumours. Based on our preclinical findings and $\beta$-lap's mechanism of action, we included assessment of tumour NQO1 expression by immunohistochemistry (IHC) as an exploratory (and subsequently enrolment) biomarker. We also analysed NQO1 genotypic polymorphisms, which are associated with the absence of NQO1 in normal tissue and may influence drug exposure, ${ }^{18,19}$ using a rapid blood assay.

\section{METHODS}

This clinical trial (NCT01502800) was approved by the University of Texas Southwestern Medical Center Institutional Review Board (IRB study number 042011-005). All subjects provided written informed consent prior to undergoing any study-related procedures. This was an open-label, single-institution, $3+3$ dose escalation phase 1 trial, initially designed to include a planned biomarker-selected expansion cohort at the maximum tolerated dose (MTD). The primary objective of the study was to determine the safety, tolerability, and MTD of ARQ 761. Secondary objectives included efficacy and pharmacokinetic (PK) analyses. Exploratory objectives included predictive and pharmacodynamic biomarkers.

\section{Patient selection}

Key inclusion criteria included the following: refractory advanced solid tumour for which standard curative or palliative measures do not exist or are no longer effective; $\geq 4$ weeks since prior chemotherapy, $\geq 3$ weeks since prior radiation therapy, $\geq 2$ weeks since prior molecularly targeted therapy (including small molecules and monoclonal antibodies); measurable disease by Response Evaluation Criteria in Solid Tumours (RECIST) version $1.1 ;{ }^{20}$ Eastern Cooperative Oncology Group performance status $0-1$; central venous access (due to local haemorrhage and infusion site necrosis upon repeated dosing in preclinical studies and intolerance of repeated peripheral dosing of ARQ 501 in earlier clinical trials); adequate clinical laboratory parameters (absolute neutrophil count $[A N C] \geq 1500 / \mu \mathrm{L}$, haemoglobin $\geq 10 \mathrm{~g} / \mathrm{dL}$ [transfusion permitted up to 7 days prior to enrolment], platelets $\geq 100,000 / \mu \mathrm{L}$, total bilirubin less than institutional upper limit of normal [ULN], aspartate aminotransferase and alanine transaminase $\leq 2.5 \times$ institutional ULN $[\leq 5 \times$ institutional ULN in the presence of liver metastases], creatinine clearance $\geq 60 \mathrm{~mL} / \mathrm{min} /$ $\left.1.73 \mathrm{~m}^{2}\right)$; and availability of 10 unstained slides or paraffinembedded tissue block from archived tumour specimen. After analysis of the first 20 patients with available efficacy and tissue biomarker data, enrolment was restricted to patients with NQO1positive tumours (defined as $H$-score $\geq 200$ ). We permitted tissue pre-screening, such that patients' NQO1 status could be determined prior to their needing initiation of new treatment. Principal exclusion criteria were untreated brain metastases, leptomeningeal disease of treatment status, pregnancy, and concurrent receipt of hepatic enzyme-inducing antiseizure drugs. There was no limit to the number of prior cancer therapies.

\section{Study treatment}

Initially, ARQ 761 monotherapy was administered as a 1-h weekly infusion. During the conduct of the trial, the emergence of frequent and clinically significant haemolytic anaemia at higher doses led to changes in infusion length and schedule, with ARQ 761 administered as a 2- or 3-h infusion weekly, every other week (QOW), or $2 / 3$ weeks. Prolongation of infusion duration was implemented after we observed more haemolytic anaemia with the $1-\mathrm{h} 390 \mathrm{mg} / \mathrm{m}^{2}$ dosing level than we did with the $195 \mathrm{mg} / \mathrm{m}^{2}$ dose. However, in a single patient treated at the $390 \mathrm{mg} / \mathrm{m}^{2}$ dose, we noted evidence of early recovery from haemolysis despite ongoing weekly dosing, when the infusion duration was prolonged from 1 to $2 \mathrm{~h}$ due to a suspected mild infusionrelated reaction. This observation persisted within the expansion cohort of $195 \mathrm{mg} / \mathrm{m}^{2}$ dose level, for which haemoglobin levels were more stable level for patients who received 2 -h infusions than those for those who received 1-h infusions. ARQ 761 was administered without premedication. Treatment was continued until disease progression or unacceptable toxicity.

The starting dose of $195 \mathrm{mg} / \mathrm{m}^{2}$ represented $50 \%$ of the MTD of ARQ 501, the earlier, insoluble formulation of $\beta$-lap that was associated with DLT attributed to high levels of the carrier molecule HP $\beta C D$. The dose was increased by $100 \%$ after the first dose cohort, then by $40 \%$ after subsequent cohorts. Intra-patient escalation from lower dose levels to successfully administered dose levels was permitted. DLT was defined as any of the following toxicities considered possibly, probably, or definitely related to ARQ 761 during the first cycle (4 weeks for weekly or biweekly regimen; 3 weeks for $2 / 3$ weeks regimen): (1) $\geq$ grade 3 haemolysis or haemolytic anaemia (transfusion for haemolysis or haemolytic anaemia was considered a DLT only if haemoglobin $<8.0 \mathrm{~g} / \mathrm{dL}$ [threshold for grade 3 anaemia]); (2) grade 4 neutropenia or thrombocytopenia, or grade 3 thrombocytopenia in the presence of bleeding; and (3) $\geq$ grade 3 non-haematological toxicity that does not spontaneously resolve within $2 \mathrm{~h}$ postinfusion. Grade 3 nausea/vomiting or diarrhoea was considered a DLT only if it occurred despite optimal medical management. Grade 3-4 hyperbilirubinaemia was considered a DLT only if it did not recover to grade 1 after 7 days. When transient grade 3 hypoxia was observed at the completion of infusion based on pulse oximetry (a surrogate marker of methemoglobinaemia) without clinically significant symptoms, it was not considered a DLT. Dose reductions were implemented for recurrent grade 3 and any grade 4 non-haematologic toxicities.

\section{Study assessments}

Safety assessments consisted of monitoring patient-reported symptoms, pre- and post-infusion vital signs, physical examination findings, blood and urine tests, and ECGs. In addition to standard blood counts and chemistries, markers of haemolysis (reticulocyte count, haptoglobin) were collected regularly. Toxicities were graded according to the $\mathrm{NCI} C \mathrm{CTCAE}$, Version 4.0. Patients were assessed for response to therapy using RECIST 1.1 every 2 cycles of therapy. In some patients with hypoxia noted on pulse oximetry suggestive of methemoglobinaemia, arterial blood gas was obtained. In the event of a change in haemoglobin related to ARQ 761 therapy, the following tests could be performed: peripheral blood film, Coomb's test (direct and indirect), 
reticulocyte count, haptoglobin, methemoglobin, bilirubin (total and direct), lactate dehydrogenase (LDH), free plasma haemoglobin, and oxygen saturation. Subjects were eligible to continue treatment if neutrophil count was $\geq 1000 / \mathrm{mL}$, platelets were $\geq 50,000 / \mathrm{mL}$, and haemoglobin $\geq 8.0 \mathrm{mg} / \mathrm{dL}$.

\section{PK studies}

Because ARQ 761 is rapidly and completely converted to ARQ 501 upon dosing, we estimated the PKs of ARQ 761 by following the plasma concentrations of ARQ 501 ( $\beta$-lap). During the first and fourth infusions of study drug, serial blood samples for determination of plasma ARQ 501 levels were drawn at the following time points: pre-infusion, infusion start, $15 \mathrm{~min}, 30 \mathrm{~min}$, $60 \mathrm{~min}, 120 \mathrm{~min}$ (for the 2 -h infusion cohort), $15 \mathrm{~min}$ post-infusion, $1 \mathrm{~h}$ post-infusion, $3 \mathrm{~h}$ post-infusion, $5 \mathrm{~h}$ post-infusion, $24 \mathrm{~h}$ postinfusion, $48 \mathrm{~h}$ post-infusion, and $168 \mathrm{~h}$ post-infusion. ARQ 501 plasma concentrations were determined using an ultra-highperformance liquid chromatography tandem mass spectrometric analytical method. This method utilised a Nexera Series UHPLC system (Shimadzu Corporation, Kyoto, Japan), which was interfaced with a Sciex QTRAP 5500 mass spectrometer (Foster City, CA, USA).

Sample preparation included a liquid-liquid extraction of ARQ 501 and the internal standard dihydrotanshone (IS) from plasma. Briefly, $100 \mu \mathrm{L}$ of a $700 \mathrm{ng} / \mathrm{mL}$ solution of IS in methanol: acetonitrile (3:1) was added to a 2-mL Eppendorf tube and spun to dryness on a CentriVap benchtop vacuum concentrator (Labconco, Kansas City, USA). To the dried tube, $25-\mu \mathrm{L}$ aliquots of plasma, $25-\mu \mathrm{L}$ of blank plasma, and $200-\mu \mathrm{L}$ de-ionised water were added and vortex mixed. To the resultant solution, a 1-mL aliquot of methyl tert-butyl ether:ethyl acetate (1:1) was added. The mixture was shaken for $10 \mathrm{~min}$ at $2500 \mathrm{rpm}$ on a VWR shaker. Samples were then centrifuged for $10 \mathrm{~min}$ at $4000 \mathrm{rpm}$ at $4.0^{\circ} \mathrm{C}$. Subsequently, $750 \mu \mathrm{L}$ of the resultant clear supernatant was transferred to a 2-mL Eppendorf tube and dried at $35^{\circ} \mathrm{C}$ for $1.5 \mathrm{~h}$ using a CentriVap benchtop vacuum concentrator for $30 \mathrm{~min}$. The dried sample was reconstituted with $300 \mu \mathrm{L}$ of acetonitrile: water (1:1). A 1- $\mu \mathrm{L}$ sample was injected onto the analytical column (Kinetex C18, $1.7 \mu \mathrm{m}, 30 \times 2.1 \mathrm{~mm}$ ), which was maintained at $40 \pm$ $2{ }^{\circ} \mathrm{C}$. Isocratic elution of ARQ 501 and internal standard was achieved using a flow rate of $0.75 \mathrm{~mL} / \mathrm{min}$, a mobile-phase composition of acetonitrile:water:formic acid (45:55:0.1), and 4min run time. Detection of ARQ 501 and internal standard was carried out in multiple-reaction monitoring mode with positive polarity, by monitoring 242.9>186.9 for ARQ 501 and 278.9> 205.2 for internal standard. Quadrupole Q1 and Q3 were set on unit resolution. The standard curve was linear $(r>0.995)$ over the concentration range of $10-2000 \mathrm{ng} / \mathrm{mL}$.

The analytical data obtained were processed by the Analyst software $^{\mathrm{TM}}$ (version 1.6.2). The PK parameters of ARQ 501 were estimated using the Phoenix WinNonLin (Certara USA, Clayton, MO, USA) software package.

Correlative studies

Methods for correlative studies, including NQO1 polymorphisms and tumour NQO1 expression, are provided in Supplemental Materials.

Statistical analysis

All patients receiving at least one dose of ARQ 761 were considered evaluable for safety analyses. Patients experiencing a DLT or those who received all scheduled doses of ARQ 761 within the DLT window were considered evaluable for DLT assessment. Patients who received at least a full dosing regimen of ARQ 761 within the DLT window and had at least one disease assessment following the initiation of therapy were considered evaluable for response.
Summary statistics for patient characteristics were reported. Adverse events were summarised in tables and presented by dose level, seriousness, severity, and relatedness. We determined the rate of disease control (partial response or stable disease) and $95 \%$ confidence intervals (Cls). We separated patients into NQO1-low and -high subgroups according to NQO1 IHC $\mathrm{H}$-score using a datadriven approach to define an objective cutoff value by using model-based clustering method implemented by "mclust" package version 4.4 for R. ${ }^{21}$ Progression-free survival (PFS) time was calculated from the date of first dose until disease progression was detected. PFS curves were estimated using the product-limit method of Kaplan-Meier with the log-rank test. We assessed the association between pharmacodynamic end points, PKs, toxicity, and clinical outcomes using Pearson correlation, linear regression, and Cox proportional hazards.

\section{RESULTS}

Patient characteristics and treatment exposure

A total of 78 patients had tumour tissue successfully tested for NQO1 expression. Tumour types and NQO1 expression levels are listed in Supplemental Table 1. This cohort included 42 patients who were accrued to the treatment study. Reasons that the other 36 patients were not enrolled to the treatment study included the following: (a) found to be ineligible; (b) did not need a new treatment initiated before the study ended; (c) no slot available; (d) clinician/patient preference; and (e) tumour tissue was NQO1negative (an exclusion factor only after institution of the enrolment biomarker). Baseline patient characteristics are presented in Table 1. Initially, we treated 5 patients at the $195 \mathrm{mg} / \mathrm{m}^{2}$ weekly 1-h infusion schedule and 4 patients at the $390 \mathrm{mg} / \mathrm{m}^{2} 1-\mathrm{h}$ infusion weekly schedule. We noted more frequent and more profound anaemia in the $390 \mathrm{mg} / \mathrm{m}^{2}$ cohort. In a single patient treated at the $390 \mathrm{mg} / \mathrm{m}^{2}$ dose level, we noted evidence of early recovery from haemolysis (despite ongoing weekly dosing) when

\begin{tabular}{ll}
$\begin{array}{l}\text { Table 1. Baseline characteristics of patients receiving ARQ } 761 \\
\text { treatment }\end{array}$ \\
\hline Characteristic & Number (\%) or median (range) \\
\hline Total patients & 42 \\
Age (years) & $65(37-86)$ \\
Sex & \\
Male & $25(60)$ \\
Female & $17(40)$ \\
Race & \\
White & $36(86)$ \\
African-American & $4(10)$ \\
Asian & $1(2)$ \\
Other & $1(2)$ \\
Primary tumour site & \\
Lung & $17(40)$ \\
Colorectal & $5(12)$ \\
Bladder & $4(10)$ \\
Pancreas & $4(10)$ \\
Breast & $2(5)$ \\
Bile duct & $2(5)$ \\
Stomach & $2(5)$ \\
Other & $6(14)$ \\
Number of lines of prior therapy & $4(1-12)$ \\
\hline
\end{tabular}

a One each of oesophageal, larynx, melanoma, Merkel cell, skin squamous cell, and thymus 
Phase 1 study of ARQ 761, a $\beta$-lapachone analogue that promotes...

\begin{tabular}{|c|c|c|c|c|c|c|c|c|c|c|}
\hline \multirow{3}{*}{$\begin{array}{l}\text { Dose } \\
N\end{array}$} & \multirow{2}{*}{\multicolumn{2}{|c|}{$\frac{195 \mathrm{mg} / \mathrm{m}^{2}}{10}$}} & \multirow{2}{*}{\multicolumn{2}{|c|}{$\frac{390 \mathrm{mg} / \mathrm{m}^{2}}{17}$}} & \multirow{2}{*}{\multicolumn{2}{|c|}{$\frac{450 \mathrm{mg} / \mathrm{m}^{2}}{11}$}} & \multirow{2}{*}{\multicolumn{2}{|c|}{$\frac{550 \mathrm{mg} / \mathrm{m}^{2}}{4}$}} & \multirow{2}{*}{\multicolumn{2}{|c|}{$\frac{\text { Total }}{42}$}} \\
\hline & & & & & & & & & & \\
\hline & Grade $1-2$ & Grade 3-4 & Grade 1-2 & Grade $3-4$ & Grade $1-2$ & Grade 3-4 & Grade $1-2$ & Grade 3-4 & Grade 1-2 & Grade 3-4 \\
\hline Anaemia & $8(80)$ & $2(20)$ & $14(82)$ & $3(18)$ & $11(100)$ & $4(36)$ & $3(75)$ & $1(25)$ & $36(86)$ & $10(24)$ \\
\hline Fatigue & $2(20)$ & $0(0)$ & $7(41)$ & $1(6)$ & $6(55)$ & $0(0)$ & $2(50)$ & $1(25)$ & $17(40)$ & $2(2)$ \\
\hline Vomiting & $1(10)$ & $1(10)$ & $2(12)$ & $1(6)$ & $0(0)$ & $0(0)$ & $2(50)$ & $0(0)$ & $5(12)$ & $2(5)$ \\
\hline Nausea & $1(10)$ & $1(10)$ & $1(6)$ & $0(0)$ & $1(9)$ & $0(0)$ & $3(75)$ & $0(0)$ & $6(14)$ & $1(2)$ \\
\hline
\end{tabular}

the infusion duration was prolonged from 1 to $2 \mathrm{~h}$ due to a suspected mild infusion-related reaction. Accordingly, we modified the treatment schedule to a 2-h infusion administered either QOW or the first 2 out of 3 weeks. At any given dose level, the infusion duration could be prolonged to $3 \mathrm{~h}$ (associated with a lower $C_{\max }$ ) if the MTD was exceeded, rather than dose deescalation.

Overall, 10 patients were treated at the $195 \mathrm{mg} / \mathrm{m}^{2}$ level (all weekly dosing; five received 1 -h infusions; five received $2-\mathrm{h}$ infusions), 17 patients at the $390 \mathrm{mg} / \mathrm{m}^{2}$ level (three 1-h infusions weekly; four 2-h infusions weekly; three 2-h infusions QOW; seven 2 -h infusions 2 out of every 3 weeks), 11 patients at the $450 \mathrm{mg} / \mathrm{m}^{2}$ level (five 2-h infusions QOW, and six 2-h infusions 2 out of every 3 weeks), and 4 patients at the $550 \mathrm{mg} / \mathrm{m}^{2}$ level (one 2-h infusion 2 out of every 3 weeks, one 2-h infusion QOW, two 3-h infusions QOW) (Supplemental Fig. 1). Across all dose levels, the median number of treatment cycles was 2 (range 1-6). After analysis of the first 20 patients with available efficacy and NQO1 biomarker data, which occurred after accrual of the first 34 patients, enrolment was restricted to those patients with NQO1-positive tumours (defined as $\mathrm{H}$-score $\geq 200$ ). Study accrual was stopped after six additional patients were enrolled because, even in this enriched subset of patients, single-agent activity of ARQ 761 appeared modest and further clinical development would focus on combination regimens. Reasons for treatment discontinuation included disease progression $(n=35)$, DLT $(n=1)$, and non-DLT toxicities $(n=6)$. One patient died while on study, which was considered related to the underlying cancer and unrelated to study drug or procedure.

Toxicity

Table 2 lists treatment-related adverse events experienced by $\geq 10 \%$ of patients according to grade and dose cohort. The principal treatment-related toxicities were anaemia, haemolysis, and hypoxia (representing possible methemoglobinaemia). Supplemental Table 2 lists principal toxicities according to grade, dose level, schedule, and duration. Across all dose levels, anaemia occurred in approximately $80 \%$ of patients (14\% grade 3 ) and generally had characteristics consistent with a haemolysis mechanism (increase in reticulocyte count, decrease in haptoglobin level). As anticipated, a less frequent infusion schedule appeared to be better tolerated, with lower rates of fatigue and high-grade anaemia in the 2/3 weeks and QOW groups than the weekly group (Supplemental Table 2), presumably due to more opportunity for recovery from treatment-related anaemia events. Other lineages (white blood cells, platelets) were essentially unaffected, making myelosuppression unlikely. Haemolytic anaemia did not appear to be immune-mediated (Coomb's test negative) and responded to transfusions. In no cases was haemolytic anaemia associated with renal dysfunction, which can arise in severe cases from renal tubular obstruction by free plasma haemoglobin. In general, we also observed improved tolerability with longer infusion time.
Although we had hypothesised that the far lower concentration of $H P \beta C D$ required for $A R Q 761$ formulation would result in less haemolytic anaemia than was seen with ARQ 501, once dose was escalated to $390 \mathrm{mg} / \mathrm{m}^{2}$, the prevalence and severity of this toxicity became apparent. To characterise further, we sought to obtain haemolysis parameters at the time of $\geq$ grade 3 anaemia $(\mathrm{Hgb}<8.0 \mathrm{~g} / \mathrm{dL}$ ) (Supplemental Table 3). Unfortunately, many of these cases have missing data because (a) we either had not started collecting the data by that point or (b) the anaemia event occurred in a setting other than our clinical trials unit. For those cases with available data at the time of grade 3 anaemia, we observed a wide range of haemolysis parameters, including bilirubin $(0.4-2.0 \mathrm{mg} / \mathrm{dL})$, LDH (165-560 units/L), haptoglobin (155-408 $\mathrm{mg} / \mathrm{dL})$, and reticulocytes (2.9-8.6\%).

Transient decrease in peripheral oxygen saturation (a surrogate marker of possible methemoglobinaemia) was noted in cases at all dose levels. At the $195 \mathrm{mg} / \mathrm{m}^{2}$ dose level, oxygen saturation decreased $4-5 \%$ during infusion, with recovery starting upon infusion completion and levels usually returning to baseline within 2-3 h. Associated symptoms were rare. At higher dose levels, oxygen saturation levels decreased $10-12 \%$ during infusion and in some cases required $24 \mathrm{~h}$ to recover. In seven cases, arterial blood gas was obtained. In all of these cases, $\mathrm{PaO}_{2}$ was normal at the time of peripheral desaturation, consistent with possible methemoglobinaemia. At higher dose levels, clinical features suggestive of possible methemoglobinaemia were noted transiently, including dizziness and mild nausea. No cases featured cyanosis or organ dysfunction. Patients did not report dyspnoea, further supporting the observation that the peripheral oxygen desaturation did not represent true hypoxemia. These apparent cases of methemoglobinaemia were not associated with organ dysfunction. Methemoglobin levels were determined in seven cases. Despite a clinical picture highly suggestive of methemoglobinaemia, the results were normal, ranging from $0.2 \%$ to $0.8 \%$ (laboratory reference range $0.4-1.5 \%$ ).

A total of five DLTs occurred at the following levels: $390 \mathrm{mg} / \mathrm{m}^{2}$ weekly $(N=3), 450 \mathrm{mg} / \mathrm{m}^{2} 2 / 3$ weeks $(N=1)$, and $550 \mathrm{mg} / \mathrm{m}^{2}$ $2 / 3$ weeks $(N=1)$. All DLTs reflected anaemia. Based on these events and consideration of the overall feasibility of long-term treatment, the MTD was considered $390 \mathrm{mg} / \mathrm{m}^{2}$ as a 2-h infusion administered every 2 weeks.

\section{Efficacy}

Of the 32 patients evaluable for radiographic response, the best response was stable disease in 12 patients (disease control rate $38 \%$ ) and progressive disease in 20 patients. Radiographic responses and duration of therapy are shown according to tumour NQO1 status in Fig. 1a, b. Minor radiographic response was noted in 6 patients, including 1 patient with heavily pretreated (five prior lines of therapy) metastatic bladder cancer (NQO1 $\mathrm{H}$-score 200) treated at dose level $195 \mathrm{mg} / \mathrm{m}^{2}$ who experienced shrinkage of multiple pulmonary metastases (Fig. 2). After analysis of 20 patients with evaluable response and available 
a

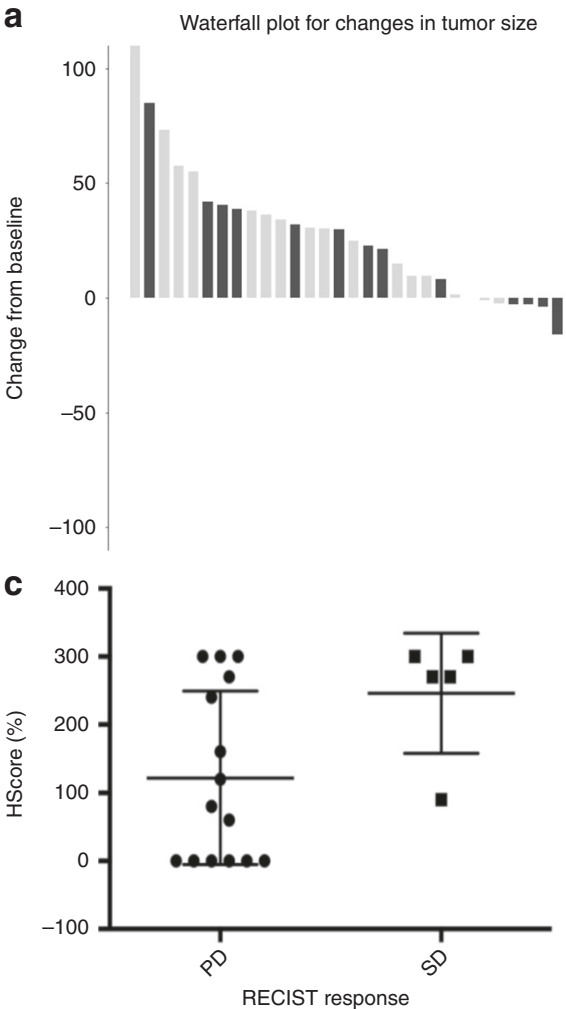

b

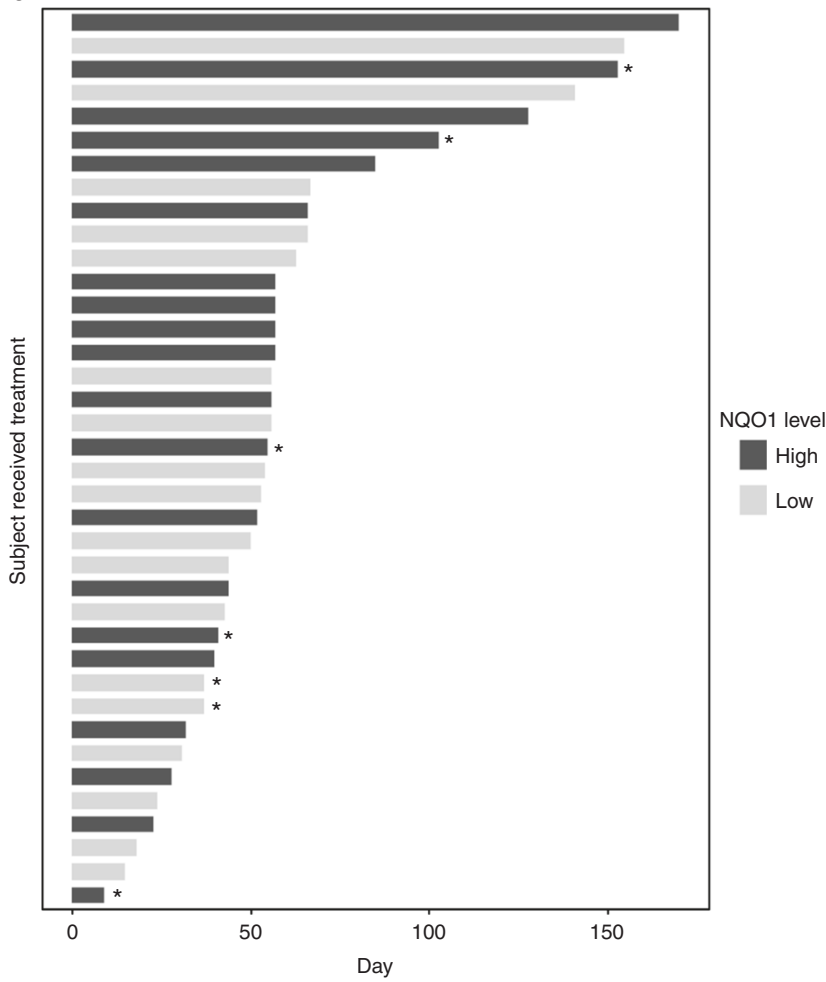

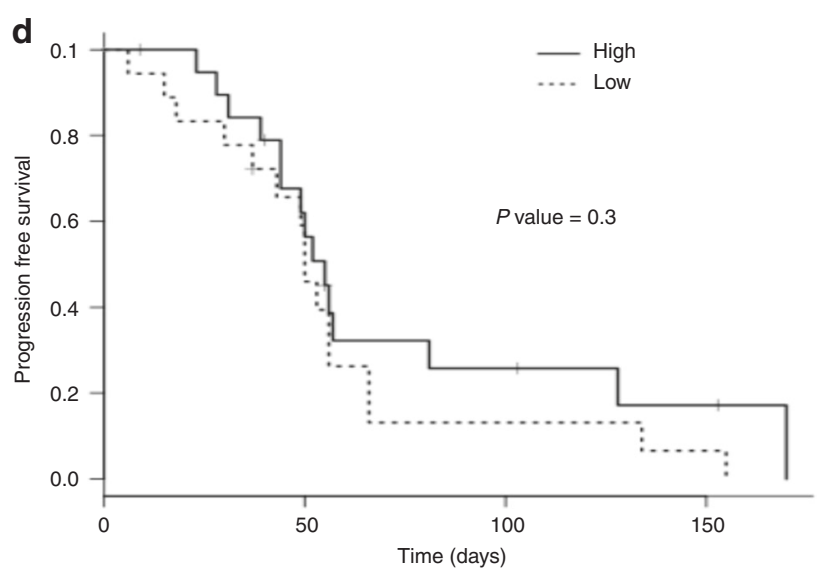

Fig. 1 Efficacy of ARQ 761 according to tumour NQO1 expression. a Waterfall plot demonstrating best radiographic response in the overall study population. Dark bars indicate NQO1-high cases; light bars indicate NQO1-low cases. b Swimmer's plot demonstrating time on therapy in the overall study population. Dark bars indicate NQO1-high cases; light bars indicate NQO1-low cases. Asterisks indicate cases for which treatment was discontinued for a reason other than disease progression. c Radiographic response among first 20 patients with both evaluable efficacy and tumour NQO1 expression data. NQO1 expression was higher among patients achieving stable disease compared to those with primary disease progression $(P=0.06)$. Of note, all six patients with NQO1-negative tumours had progressive disease. Based on these data, subsequent enrolment was limited to patients with NQO1-positive tumours (defined as $H$-score $\geq 200$ ). PD progressive disease, RECIST, Response Evaluation Criteria in Solid Tumours, SD stable disease. d Progression-free survival according to tumour NQO1 expression. HR 0.68; 95\% Cl, 0.33-1.39; $P=0.3$

NQO1 expression level, we observed a near significant association $(P=0.06)$ between tumour NQO1 expression $(H$-score $\geq 200)$ and response (Fig. 1C). Of note, no NQO1-negative ( $H$-score 0$)$ cases $(N=6)$ achieved disease control. Based on these observations, the protocol was amended to limit enrolment to NQO1-positive cases $(H$-score $\geq 200)$, following which an additional 8 patients were treated. PFS according to NQO1 level for the entire study population is shown in Fig. 1d. Four-month PFS rate was $26 \%$ in NQO1-high tumours vs. $13 \%$ in NQO1-low tumours $(P=0.3)$.
Pharmacokinetics

Across all dose and schedule cohorts, PK data were available for a total of 27 patients in the Dose 1 (first dose) group and a total of 17 patients in the Dose 4 (fourth dose) group. PKs of ARQ 761 were estimated using the concentration vs. time profiles of ARQ 501. The results of these PK determinations demonstrated high levels of patient variability. PKs were generally characterised by a rapid initial distribution and a slower terminal elimination; therefore, a two-compartment model was used to estimate the PKs. 

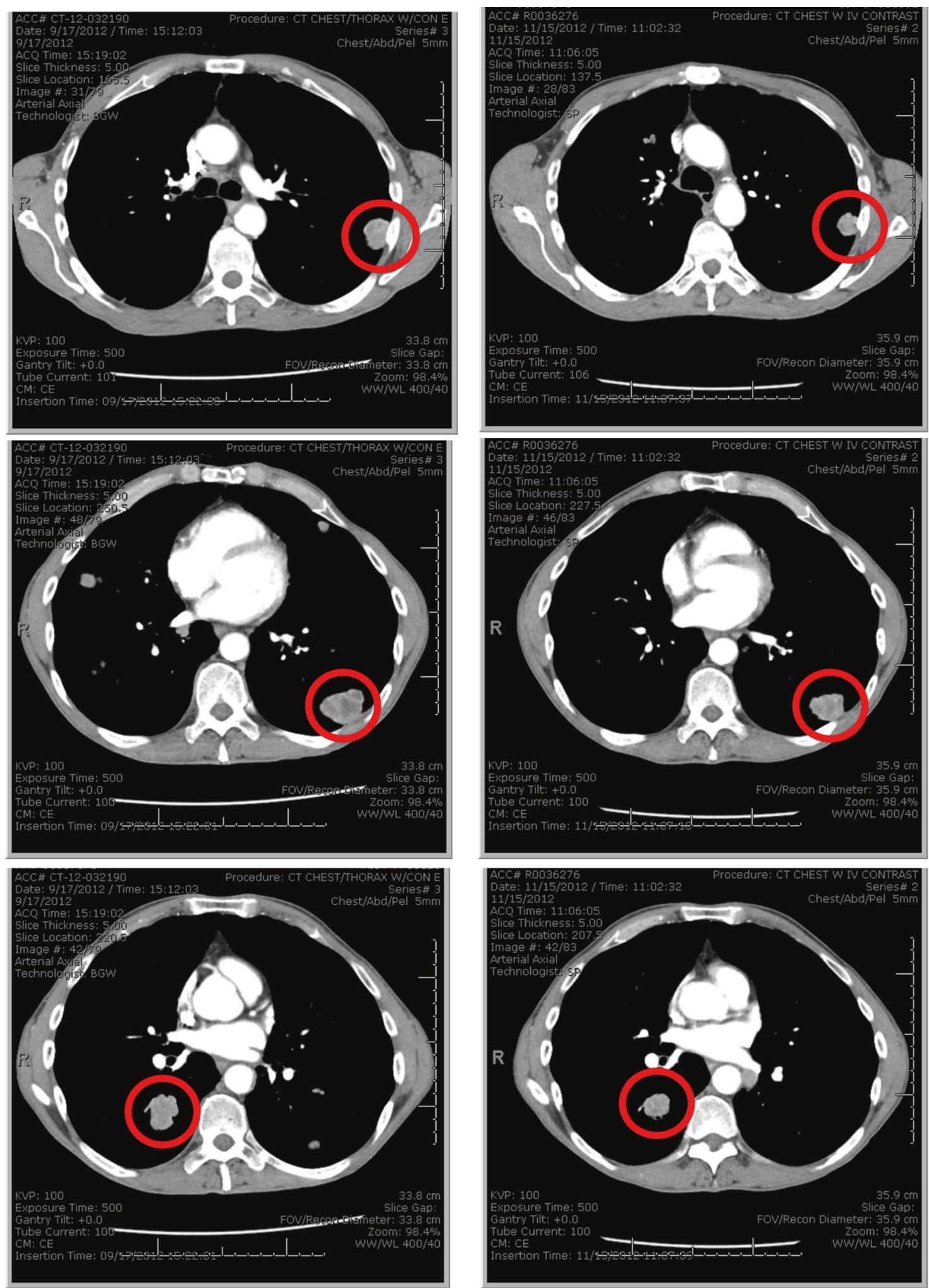

Fig. 2 Example of minor radiographic response from ARQ 761. Patient had heavily pre-treated bladder cancer (five prior lines of therapy). Images show multiple pulmonary metastases that decreased in size after initiation of study treatment

The determined PK parameters for Dose 1 are presented in Table 3A and Dose 4 are presented in Table 3B. The use of a 2-h vs. 1 - $h$ infusion in Dose 1 resulted in a lower observed $C_{\max }$, which correlated well with the better tolerability of the 2-h infusions (Supplemental Table 2). For instance, in the $195 \mathrm{mg} / \mathrm{m}^{2}$ weekly dose cohort, the rate of anaemia was $100 \%$ (40\% grade $3-4)$ with a 1-h infusion, compared to $60 \%$ (0\% grade 3-4) with a 2-h infusion. The observed high variability of the area under the receiver operating characteristic curve (AUC) makes statistically significant clinical correlations difficult; however, the AUC did generally increase with increased dose. The half-life as measured as the halflife of elimination from the central compartment of the two- 
compartment model was fairly consistent among the dose levels and dosing regimen. PKs for Dose 1 vs. Dose 4 demonstrated that ARQ 501 does not accumulate to any appreciable extent using this dosing regimen. Within specific dose levels, a potential

Table 3A. Dose 1 pharmacokinetic parameters of plasma $\beta$-lapachone following intravenous administration of ARQ 761

\begin{tabular}{llllll}
\hline $\begin{array}{l}\text { Dose } \\
\left(\mathrm{mg} / \mathrm{m}^{2}\right)\end{array}$ & $\begin{array}{l}\text { Infusion } \\
\text { time }\end{array}$ & $N$ & $\begin{array}{l}\mathrm{AUC}(0-\text { last }) \\
(\mathrm{ng} \times \mathrm{h} / \mathrm{mL})\end{array}$ & $\begin{array}{l}C_{\max } \\
(\mathrm{ng} / \mathrm{mL})\end{array}$ & $\begin{array}{l}\text { Half-life } \\
(\mathrm{h})^{\mathrm{a}}\end{array}$ \\
\hline 195 & $1 \mathrm{~h}$ & 4 & $10,463 \pm 6103$ & $1294 \pm 258$ & $1.8 \pm 1.0$ \\
195 & $2 \mathrm{~h}$ & 3 & $23,369 \pm 15,1287$ & $947 \pm 233$ & $3.1 \pm 2.0$ \\
390 & $1 \mathrm{~h}$ & 4 & $23,910 \pm 20,745$ & $2418 \pm 705$ & $1.2 \pm 0.9$ \\
390 & $2 \mathrm{~h}$ & 13 & $33,150 \pm 27,468$ & $1798 \pm 738$ & $2.6 \pm 1.5$ \\
450 & $2 \mathrm{~h}$ & 10 & $21,276 \pm 13,323$ & $1652 \pm 442$ & $1.5 \pm 1.4$ \\
\hline
\end{tabular}

aHalf-life of elimination from the central compartment of the twocompartment model

Table 3B. Dose 4 pharmacokinetic parameters of plasma $\beta$-lapachone following intravenous administration of ARQ 761

\begin{tabular}{llllll}
\hline $\begin{array}{l}\text { Dose } \\
\left(\mathrm{mg} / \mathrm{m}^{2}\right)\end{array}$ & $\begin{array}{l}\text { Infusion } \\
\text { time }\end{array}$ & $\mathrm{N}$ & $\begin{array}{l}\mathrm{AUC}_{(0-\text { last }} \\
(\mathrm{ng} \times \mathrm{h} / \mathrm{mL})\end{array}$ & $\begin{array}{l}\mathrm{C}_{\max } \\
(\mathrm{ng} / \mathrm{mL})\end{array}$ & $\begin{array}{l}\text { Half-life } \\
(\mathrm{h})^{\mathrm{a}}\end{array}$ \\
\hline 195 & $1 \mathrm{~h}$ & 3 & $30,698 \pm 9152$ & $1540 \pm 464$ & $4.7 \pm 1.7$ \\
195 & $2 \mathrm{~h}$ & 4 & $32,007 \pm 16,793$ & $968 \pm 63$ & $3.5 \pm 1.9$ \\
390 & $1 \mathrm{~h}$ & 2 & $20,374 \pm 7775$ & $2785 \pm 754$ & $1.6 \pm 0.3$ \\
390 & $2 \mathrm{~h}$ & 3 & $41,537 \pm 33,968$ & $3215 \pm$ & $2.5 \pm 2.0$ \\
& $2 \mathrm{~h}$ & 9 & $26,699 \pm 14,251$ & $2321 \pm$ & $2.4 \pm 2.4$ \\
450 & 2 & & & 1339 & \\
\hline
\end{tabular}

a Half-life of elimination from the central compartment of the twocompartment model relationship between certain PK parameters and clinical effects was observed. These relationships included a trend toward correlation between Vss = apparent volume of distribution at steady state and PFS and a correlation between AUC and disease control $(P=0.03)$. There were no PK/pharmacodynamic relationships determined because pharmacodynamic end points were not included in the study.

Biomarker studies

We successfully determined tumour NQO1 expression by IHC in all enrolled cases (Fig. 3). Once enrolment was limited to NQO1positive cases, we developed a CLIA-certified IHC assay with a turnaround time of $\leq 72 \mathrm{~h}$ permitting real-time assessment of potential study candidates. Germline NQO1 polymorphisms were assessed in all enrolled patients. All cases were NQO1*1.

\section{DISCUSSION}

This study is a first-in-human phase 1 trial of ARQ 761, a synthetic analogue of the NQO1-bioactivatable drug $\beta$-lap that is administered intravenously through central access. In this trial, we observed haemolytic anaemia as a DLT, with an MTD and recommended phase 2 dose of $390 \mathrm{mg} / \mathrm{m}^{2}$ as a 2-h infusion QOW. At this level, patients also experienced largely asymptomatic possible methemoglobinaemia, manifest as transient decrease in peripheral oxygen saturation without a concomitant decrease in $\mathrm{PaO}_{2}$. As we had hypothesised, ARQ 761 appeared to exert antitumour effects in an NQO1-dependent fashion. All efficacy parameters (response, PFS, time on study treatment) had either a statistically significant (or trend towards) association with tumour NQO1 expression. Although no patients experienced formal partial response by RECIST parameters, approximately $20 \%$ of cases had some evidence of radiographic shrinkage, including at the lowest dose level tested.

Compared to the previous formulation, ARQ 501, ARQ 761 requires only $5 \%$ the total $\mathrm{HP} \beta C D$ - the carrier molecule thought to be the aetiology of haemolysis in preclinical models. Nevertheless, as with ARQ 501, an apparent haemolytic anaemia
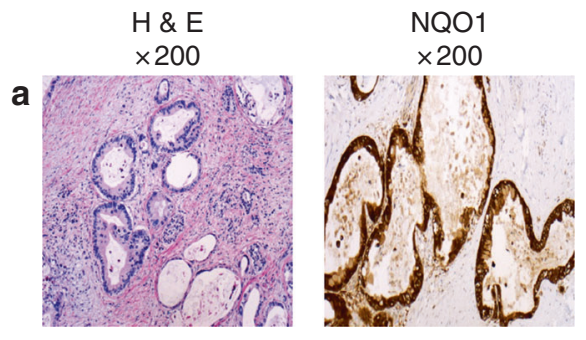

b
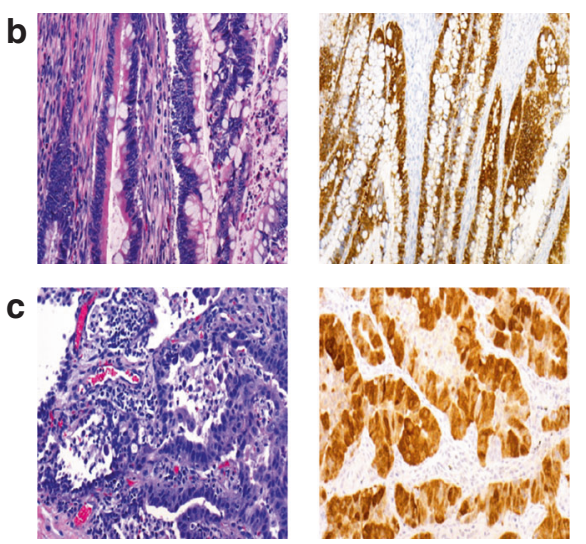
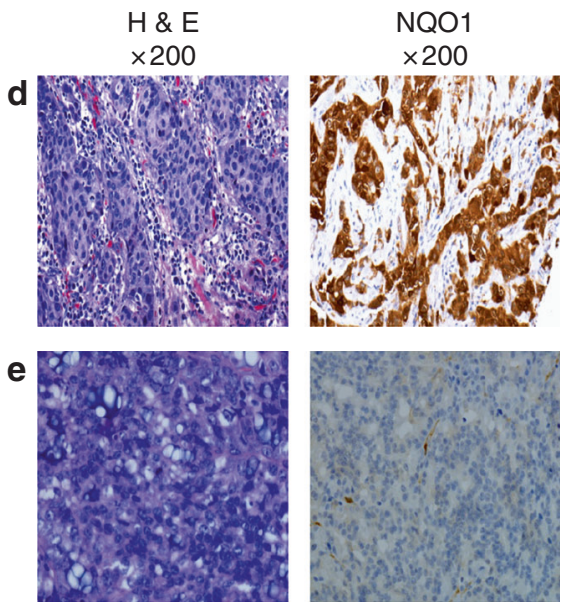

Fig. 3 NQO1 IHC staining. a Moderately differentiated pancreatic adenocarcinoma, 3+ NQO1 staining, $H$-score 300 . b Moderately differentiated colonic adenocarcinoma, 3+ NQO1 staining, $\mathrm{H}$-score 250. c Lung adenocarcinoma, 3+ NQO1 staining, $\mathrm{H}$-score 290 . d Highgrade invasive ductal breast carcinoma, $3+$ NQO1 staining, $H$-score 300 . e Prostate adenocarcinoma, $0+$ NQO1 staining, $H$-score 0 
emerged as the principal toxicity and DLT in the present trial. The characterisation of the observed anaemia is challenging. The known mechanism of $\beta$-lap, as well as the lack of leukopenia and thrombocytopenia in this trial, make bone marrow suppression unlikely. Instead, separate from direct effects of the HP $\beta C D, A R Q$ 761-mediated changes in the redox state may account for this toxicity. While $\beta$-lap is a very good substrate for the two-electron oxidoreductase, NQO1, it is also an excellent substrate for oneelectron oxidoreductases, such as the b5R enzyme. ${ }^{1}$ In particular, the toxic effects of $\beta$-lap may be due to its interactions with b5R, which is expressed in the blood of mammals, where b5R is responsible for reducing haemoglobin. In the presence $\beta$-lap, b5R fails to reduce methemaglobin and results in more one-electron oxidoreductions that further oxidise the haeme molecules, contributing to methemoglobinaemia. Subsequently, it is possible that methemoglobinaemia itself drove the haemolytic events, as has been reported with other therapeutic agents, such as rasburicase. ${ }^{22}$ Although possible methemoglobinaemia was clinically evident in approximately $30 \%$ of patients (and was suspected in essentially all patients treated at higher-dose levels), methemoglobin levels were not elevated. This phenomenon may reflect challenges in the performance of this assay, which is subject to variations according to timing and handling of sample, and may also be influenced by haemolysis in the specimen. ${ }^{23}$ Furthermore, in animal models, $\beta$-lap-induced methemoglobinaemia lasted only $5 \mathrm{~min}$, so the event may be too transient for clinical detection. ${ }^{24}$ Without reliable methemoglobin levels in our study population for guidance, we consider the observed ARQ 761-associated possible methemoglobinaemia to be relatively mild given the transient nature of desaturation by pulse oximetry and the absence of cyanosis. Certainly, we have little suspicion that patients experienced life-threatening events (which occur at methemoglobin levels $>70 \%$ ). One possible approach to mitigating methemoglobinaemia is the administration of antioxidants. However, it is not known whether such a strategy would reduce therapeutic anticancer effects. In any case, although ARQ 761 does not appear to cause myelosuppression, the potential for overlapping anaemia toxicity will necessitate careful consideration when this agent is combined with cytotoxic therapies.

ARQ 761 PKs demonstrated high variability, which may in part reflect the small number of patients in many of the dosage/ schedule/infusion time groups. Additionally, the PK profile of prodrugs is generally more variable when compared to the parent drug because of inter-patient differences in the rate of conversion from prodrug to parent drug (ARQ 501). This highly variable PK profile may complicate ARQ 761 administration; agents with high variability tend to have higher adverse event rates because they often cross the adverse event concentration threshold.

This study is the first human clinical trial study to suggest an association between $\beta$-lap efficacy and positive expression of NQO1 in tumours. Pharmacologically, NQO1 has a role distinct from typical "targets" of small molecules and monoclonal antibodies. Instead of driving cancer cell survival pathways that can be inhibited by targeted therapies (e.g. epidermal growth factor receptor, vascular endothelial growth factor receptor), NQO1 activates ARQ 761 to exert cytotoxic effects via oxidative stress. Consistent with earlier reports, ${ }^{25-30}$ we observed high rates and levels of NQO1 expression in NSCLC and gastrointestinal malignancies. In contrast, only one of the three neuroendocrine lung cancers expressed NQO1, consistent with earlier reports. ${ }^{31,32}$ As expected for Western populations, we identified no *2 or *3 NQO1 genotypic polymorphisms in this study cohort. Such variants could influence NQO1 tissue expression and activity, and they may need to be considered in clinical trials in East Asian groups, in which NQO1 polymorphisms occur in up to $13 \%$ of individuals. ${ }^{33}$

Although this phase 1 trial suggests that tumours with high expression of NQO1 by IHC may be associated with clinical benefit of ARQ 761 and other $\beta$-lap analogues, from this relatively small sample it is not clear that this assay or the selected cut point $(H$ score 200) represents the optimal enrollment biomarker for $\beta$-lap trials. Most of the tissue samples analysed for NQO1 expression came from archival specimens. It is possible that NQO1 expression may have changed during the intervening lines of therapy, as preclinical models have demonstrated that ionising radiation and multiple cytotoxic therapies may induce NQO1. ${ }^{34,35}$ Furthermore, biopsy specimens have limited tumour tissue that may not reflect potential intratumoural heterogeneity. Based on preclinical studies, we hypothesised that a combination of elevated NQO1 and low catalase (a detoxifying enzyme) in tumour specimensreported as an elevated NQO1/catalase ratio-would provide the most meaningful predictor of efficacy. ${ }^{36}$ However, we were not able to reliably quantify tissue catalase expression in the available clinical samples. The observed PK parameters further complicate these considerations, as it appeared that plasma ARQ 761 levels may vary substantially within a given dose level and could be associated with clinical benefit. Unfortunately, to date, we have not developed a clinically available non-invasive pharmacodynamic biomarker for $\beta$-lap. Although this study did not include onstudy biopsies, the ongoing trial of ARQ 761 in combination with gemcitabine plus Nab-paclitaxel (NCT02514031) does incorporate post-treatment biopsies. These will allow serial monitoring of intratumoural PARYlated PARP, which correlates with antitumour effects in preclinical models. ${ }^{1,37}$ Imaging of hyperpolarised glucose or pyruvate represents another potential pharmacodynamic biomarker. ${ }^{30}$ Finally, this single-arm phase 1 study does not itself provide evidence that NQO1 expression is predictive rather than prognostic. However, NQO1 expression has been associated with worse outcomes in multiple tumour types. ${ }^{38-41}$ Thus the trend towards improved outcomes in NQO1-high cases in this trial seems likely to reflect treatment effects.

In conclusion, ARQ 761 is a synthetic analogue and prodrug of the NQO1-bioactivatable drug $\beta$-lapachone. In this phase 1 trial, ARQ 761 demonstrated highly variable PKs and possible methemoglobinaemia and haemolytic anaemia emerged as the principal toxicities. Although we had hypothesised that its formulation would mitigate key adverse effects, the ARQ 761 MTD of $390 \mathrm{mg} /$ $\mathrm{m}^{2}$ was identical to that of the earlier ARQ 501 compound. As monotherapy, ARQ 761 had only modest efficacy, resulting in minor radiographic responses in approximately $20 \%$ of patients but no partial responses. Consistent with preclinical models, this effect may be associated with tumours that have high NQO1 expression, but further biomarker development and PK profiling will be required to identify optimal populations for this treatment strategy. Recently, in vitro and in vivo studies have demonstrated synergistic effects when $\beta$-lap is combined with other treatments such as PARP inhibitors, ionising radiation, alkylating agents, glutamine metabolism inhibitors, and nicotinamide phosphoribosyltransferase inhibitors. ${ }^{27,28,37,42,43}$ Clinical evaluation of such combinations may be warranted, with careful attention to the potential for overlapping toxicity. Combination studies with chemotherapy (gemcitabine and Nab-paclitaxel for pancreatic cancer; NCT02514031) and olaparib (refractory advanced solid tumours; NCT03575078) are underway. Emerging alternative drug formulations, such as controlled release micelles and intratumoural polymer implants, $6,8,26$ may improve the tolerability of this treatment strategy. Alternatively, NQO1-bioactivatable drugs that are not good substrates for one-electron oxidoreductases (e.g. b5R) -including deoxynyboquinone and derivatives such as isobutyldeoxynyboquinone ${ }^{24}$-may mitigate the systemic redox changes that may underlie the key toxicities of ARQ 761 .

\section{ACKNOWLEDGEMENTS}

The authors thank Ms. Dru Gray for assistance with manuscript preparation. This work was supported in part by a National Cancer Institute at the National Institutes of Health, Midcareer Investigator Award in Patient-Oriented Research (K24CA201543-01; 
to D.E.G.), a National Cancer Institute Cancer Clinical Investigator Team Leadership Award (1P30 CA142543-01 supplement; to D.E.G.), the Harold C. Simmons Comprehensive Cancer Center at the University of Texas Southwestern Medical Center (1P30 CA142543-03), and ArQule, Inc. Research reported in this publication was supported in part by the Harold C. Simmons Comprehensive Cancer Center's Biomarker Research Core and Biostatistics and Bioinformatics Shared Resource, which are supported by NCl Cancer Center Support Grant 1P30 CA142543-03. Additionally, research reported in this publication was supported in part by Cancer Prevention Research Institute of Texas Core Facilities Support Award (RP170003; to W.C.P.).

\section{AUTHOR CONTRIBUTIONS}

D.E.G., F.F., and O.F. drafted the manuscript. F.F., C.C.M., R.R.K., I.S., Y.D., and V.S. performed the experiments and data analysis. R.D.L., W.C.P., V.S., O.F., X.L., Y.X., and D.E.G. performed data interpretation. All authors have contributed to conception and design of the study and are involved in the critical revision and final approval of the manuscript.Data availabilityThe data sets generated during and/or analysed during the current study are available from the corresponding author on reasonable request.

\section{ADDITIONAL INFORMATION}

Supplementary information is available for this paper at https://doi.org/10.1038/ s41416-018-0278-4.

Ethics approval and consent to participate: This study was approved by the UT Southwestern Institutional Review Board (IRB \# STU 042011-005). All patients provided informed consent. The study was performed in accordance with the Declaration of Helsinki.

Competing interests: D.E.G. has received research funding from ArQule, Inc. B.S. is an employee of ArQule, Inc. The other authors declare no competing interests.

Note: This work is published under the standard license to publish agreement. After 12 months the work will become freely available and the license terms will switch to a Creative Commons Attribution 4.0 International (CC BY 4.0).

\section{REFERENCES}

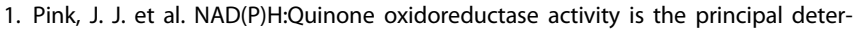
minant of beta-lapachone cytotoxicity. J. Biol. Chem. 275, 5416-5424 (2000).

2. Bentle, M. S., Bey, E. A., Dong, Y., Reinicke, K. E. \& Boothman, D. A. New tricks for old drugs: the anticarcinogenic potential of DNA repair inhibitors. J. Mol. Histol. 37, 203-218 (2006).

3. Marin, A. et al. DT-diaphorase and cytochrome B5 reductase in human lung and breast tumours. Br. J. Cancer 76, 923-929 (1997).

4. Belinsky, M. \& Jaiswal, A. K. NAD(P)H:quinone oxidoreductase1 (DT-diaphorase) expression in normal and tumor tissues. Cancer Metastasis Rev. 12, 103-117 (1993).

5. Ough, M. et al. Efficacy of beta-lapachone in pancreatic cancer treatment: exploiting the novel, therapeutic target NQO1. Cancer Biol. Ther. 4, 95-102 (2005).

6. Dong, Y. et al. Intratumoral delivery of beta-lapachone via polymer implants for prostate cancer therapy. Clin. Cancer Res. 15, 131-139 (2009).

7. Bey, E. A. et al. An NQO1- and PARP-1-mediated cell death pathway induced in non-small-cell lung cancer cells by beta-lapachone. Proc. Natl Acad. Sci. USA 104, 11832-11837 (2007).

8. Blanco, E. et al. Beta-lapachone micellar nanotherapeutics for non-small cell lung cancer therapy. Cancer Res. 70, 3896-3904 (2010).

9. Li, C. J., Li, Y. Z., Pinto, A. V. \& Pardee, A. B. Potent inhibition of tumor survival in vivo by beta-lapachone plus taxol: combining drugs imposes different artificial checkpoints. Proc. Natl Acad. Sci. USA 96, 13369-13374 (1999).

10. Park, H. J. et al. Susceptibility of cancer cells to beta-lapachone is enhanced by ionizing radiation. Int. J. Radiat. Oncol. Biol. Phys. 61, 212-219 (2005).

11. Doll, D. C., Weiss, R. B. \& Issell, B. F. Mitomycin: ten years after approval for marketing. J. Clin. Oncol. 3, 276-286 (1985)

12. Schellens, J. $H$. et al. Phase I and pharmacologic study of the novel indoloquinone bioreductive alkylating cytotoxic drug E09. J. Natl Cancer Inst. 86, 906-912 (1994).

13. Khong, H. T. et al. A phase 2 study of ARQ 501 in combination with gemcitabine in adult patients with treatment naive, unresectable pancreatic adenocarcinoma. J. Clin. Oncol. 25(18S (June 20 Supplement)), 15017 (2007).

14. Kawecki, A. et al. A phase II study of ARQ 501 in patients with advanced squamous cell carcinoma of the head and neck. J. Clin. Oncol. 25(18S (June 20 Supplement)), 16509 (2007).

15. Nasongkla, N. et al. Enhancement of solubility and bioavailability of beta-lapachone using cyclodextrin inclusion complexes. Pharm. Res. 20, 1626-1633 (2003).
16. Blanco, E. et al. Beta-lapachone-containing PEG-PLA polymer micelles as novel nanotherapeutics against NQO1-overexpressing tumor cells. J. Control. Release 122, 365-374 (2007).

17. Li, Y., Sun, X., LaMont, J. T., Pardee, A. B. \& Li, C. J. Selective killing of cancer cells by beta -lapachone: direct checkpoint activation as a strategy against cancer. Proc. Natl Acad. Sci. USA 100, 2674-2678 (2003).

18. Phillips, R. M., Burger, A. M., Fiebig, H. H. \& Double, J. A. Genotyping of NAD(P)H: quinone oxidoreductase (NQO1) in a panel of human tumor xenografts: relationship between genotype status, NQO1 activity and the response of xenografts to Mitomycin C chemotherapy in vivo(1). Biochem. Pharmacol. 62, 1371-1377 (2001).

19. Kim, S. et al. Pharmacokinetics and tolerability of MB12066, a beta-lapachone derivative targeting $\mathrm{NAD}(\mathrm{P}) \mathrm{H}$ : quinone oxidoreductase 1: two independent, double-blind, placebo-controlled, combined single and multiple ascending dose first-in-human clinical trials. Drug Des. Dev. Ther. 11, 3187-3195 (2017).

20. Eisenhauer, E. A. et al. New response evaluation criteria in solid tumours: revised RECIST guideline (version 1.1). Eur. J. Cancer 45, 228-247 (2009).

21. Fraley, C. \& Raftery, A. E. Model-based clustering, discriminant analysis, and desnity estimation. J. Am. Stat. Assoc. 97, 611-631 (2002).

22. Borinstein, S. C., Xu, M. \& Hawkins, D. S. Methemoglobinemia and hemolytic anemia caused by rasburicase administration in a newly diagnosed child with Burkitt lymphoma/leukemia. Pediatr. Blood Cancer 50, 189 (2008).

23. Cortazzo, J. A. \& Lichtman, A. D. Methemoglobinemia: a review and recommendations for management. J. Cardiothorac. Vasc. Anesth. 28, 1043-1047 (2014).

24. Huang, X. et al. An NQO1 substrate with potent antitumor activity that selectively kills by PARP1-induced programmed necrosis. Cancer Res. 72, 3038-3047 (2012).

25. Chakrabarti, G. et al. Tumor-selective use of DNA base excision repair inhibition in pancreatic cancer using the NQO1 bioactivatable drug, beta-lapachone. Sci. Rep. 5, 17066 (2015).

26. Ma, X. et al. Esterase-activatable beta-lapachone prodrug micelles for NQO1targeted lung cancer therapy. J. Control. Release 200, 201-211 (2015).

27. Moore, Z. et al. NAMPT inhibition sensitizes pancreatic adenocarcinoma cells to tumor-selective, PAR-independent metabolic catastrophe and cell death induced by beta-lapachone. Cell Death Dis. 6, e1599 (2015).

28. Chakrabarti, G. et al. Targeting glutamine metabolism sensitizes pancreatic cancer to PARP-driven metabolic catastrophe induced by ss-lapachone. Cancer Metab. 3, 12 (2015).

29. Madajewski, B., Boatman, M. A., Chakrabarti, G., Boothman, D. A. \& Bey, E. A. Depleting tumor-NQO1 potentiates anoikis and inhibits growth of NSCLC. Mol. Cancer Res. 14, 14-25 (2016).

30. Silvers, M. A. et al. The NQO1 bioactivatable drug, beta-lapachone, alters the redox state of $\mathrm{NQO} 1+$ pancreatic cancer cells, causing perturbation in central carbon metabolism. J. Biol. Chem. 292, 18203-18216 (2017).

31. Kepa, J. K. \& Ross, D. DT-diaphorase activity in NSCLC and SCLC cell lines: a role for fos/jun regulation. Br. J. Cancer 79, 1679-1684 (1999).

32. Kepa, J. K. \& Ross, D. Differential expression of the antioxidant response element within the hNQO1 promoter in NSCLC versus SCLC. Biochem. Biophys. Res. Commun. 311, 446-453 (2003).

33. Gaedigk, A. et al. NAD(P)H:quinone oxidoreductase: polymorphisms and allele frequencies in Caucasian, Chinese and Canadian Native Indian and Inuit populations. Pharmacogenetics 8, 305-313 (1998).

34. Boothman, D. A., Meyers, M., Fukunaga, N. \& Lee, S. W. Isolation of X-ray-inducible transcripts from radioresistant human melanoma cells. Proc. Natl Acad. Sci. USA 90, 7200-7204 (1993).

35. Valerio, L. G. Jr., Kepa, J. K., Pickwell, G. V. \& Quattrochi, L. C. Induction of human $\mathrm{NAD}(\mathrm{P}) \mathrm{H}$ :quinone oxidoreductase (NQO1) gene expression by the flavonol quercetin. Toxicol. Lett. 119, 49-57 (2001).

36. Bey, E. A. et al. Catalase abrogates beta-lapachone-induced PARP1 hyperactivationdirected programmed necrosis in NQO1-positive breast cancers. Mol. Cancer Ther. 12, 2110-2120 (2013).

37. Huang, $X$. et al. Leveraging an NQO1 bioactivatable drug for tumor-selective use of poly(ADP-ribose) polymerase inhibitors. Cancer Cell 30, 940-952 (2016).

38. Yang, Y. et al. Clinical implications of high NQO1 expression in breast cancers. J. Exp. Clin. Cancer Res. 33, 14 (2014).

39. Li, Z. et al. NQO1 protein expression predicts poor prognosis of non-small cell lung cancers. BMC Cancer 15, 207 (2015).

40. Cui, X. et al. High expression of NQO1 is associated with poor prognosis in serous ovarian carcinoma. BMC Cancer 15, 244 (2015).

41. Lin, L. et al. Prognostic implication of NQO1 overexpression in hepatocellular carcinoma. Hum. Pathol. 69, 31-37 (2017).

42. Park, M. T. et al. beta-lapachone significantly increases the effect of ionizing radiation to cause mitochondrial apoptosis via JNK activation in cancer cells. PLOS ONE 6, e25976 (2011).

43. Zhang, L. et al. beta-Lapachone and paclitaxel combination micelles with improved drug encapsulation and therapeutic synergy as novel nanotherapeutics for NQO1-targeted cancer therapy. Mol. Pharm. 12, 3999-4010 (2015). 